\title{
ON THE COHOMOLOGY OF ASSOCIATIVE ALGEBRAS AND LIE ALGEBRAS
}

\author{
ROLF FARNSTEINER
}

\begin{abstract}
This paper presents a sufficient condition for the vanishing of the cohomology groups of an associative algebra.
\end{abstract}

0. Introduction. In this paper, we shall present a vanishing theorem for the cohomology groups (cf. [6]) of an associative $F$-algebra.

The theory of derived functors, set forth by Cartan and Eilenberg in [2], links the cohomology groups of a Lie algebra $L$ to the cohomology groups of its universal enveloping algebra. We shall use this fact in order to interpret our general result within the framework of Lie algebra cohomology. Since its initiation by the Whitehead Lemmas $[\mathbf{8}, \mathbf{9}]$, this branch of cohomology theory has enjoyed considerable attention. However, the customary methods exclusively employed the description of Lie algebra cohomology as given in $[3]$. This had the effect that the proofs of vanishing theorems necessitated additional assumptions concerning the behavior of suitably chosen elements $[\mathbf{3}, \mathbf{5}]$. Our present result does not require such considerations and generalizes the results alluded to above.

The author wishes to acknowledge his indebtedness to G. P. Hochschild. Aside from providing kind advice concerning the exposition of this paper, he has also contributed to the final formulation of Proposition 1.1, which amounts to a clarification and simplification of the author's original proof of the vanishing theorem.

1. The main result. Throughout this section, $A$ is assumed to be an associative algebra with unity over a field $F$. Let $M$ be a two sided $A$-module, i.e. an $F$-vector space together with two module operations (left and right) such that

$$
a \cdot(m \cdot b)=(a \cdot m) \cdot b \quad \forall a, b \in A \forall m \in M .
$$

We denote by $C^{n}(A, M)$ the $F$-space of $(n-F)$-linear mappings from the $n$-fold Cartesian product of $A$ into $M(n \geq 1)$. We put $C^{0}(A, M)=M$ and $C^{n}(A, M)=0$ for $n<0$. On the graded space $C(A, M):=\bigoplus_{i \in Z} C^{i}(A, M)$ one defines a linear map $\delta$ of degree 1 by

$$
\begin{aligned}
\delta(f)\left(a_{0}, \ldots, a_{n}\right)= & a_{0} \cdot f\left(a_{1}, \ldots, a_{n}\right)+\sum_{i=1}^{n}(-1)^{i} f\left(a_{0}, \ldots, a_{i-1} a_{i}, \ldots, a_{n}\right) \\
& +(-1)^{n+1} f\left(a_{0}, \ldots, a_{n-1}\right) \cdot a_{n} ; \quad f \in C^{n}(A, M), n \geq 0 .
\end{aligned}
$$

Then $\delta^{2}=0$ and $H^{n}(A, M):=\operatorname{ker} \delta \cap C^{n}(A, M) / \operatorname{im} \delta \cap C^{n-1}(A, M)$ for $n \geq 0$ is called the $n$th cohomology group of $A$ with coefficients in $M$ (cf. [6]).

Received by the editors January 22, 1986.

1980 Mathematics Subject Classification (1985 Revision). Primary 16A61, 17B56. 
For each element $a \in A$ we define two linear maps $\gamma_{a}, \mu_{a}: C(A, M) \rightarrow C(A, M)$ of degrees -1 and 0 , respectively:

$$
\begin{aligned}
\gamma_{a}(g)\left(a_{1}, \ldots, a_{n}\right)=\sum_{j=0}^{n}(-1)^{j} g\left(a_{1}, \ldots, a_{j}, a, a_{j+1}, \ldots, a_{n}\right), g \in C^{n+1}(A, M), & n \geq 0, \\
\mu_{a}(f)\left(a_{1}, \ldots, a_{n}\right)= & a \cdot f\left(a_{1}, \ldots, a_{n}\right)+\sum_{i=1}^{n} f\left(a_{1}, \ldots, a_{i-1},\left[a_{i}, a\right], \ldots, a_{n}\right) \\
& -f\left(a_{1}, \ldots, a_{n}\right) \cdot a, \quad f \in C^{n}(A, M), n \geq 0,
\end{aligned}
$$

where $[a, b]=a b-b a \forall a, b \in A$.

The following result, which yields our vanishing theorem, is interesting in its own right. ${ }^{1}$

PROPOSITION 1.1. $\delta \circ \gamma_{a}+\gamma_{1} \circ \delta=\mu_{a}$.

PROOF. For $n<0$ there is nothing to be shown. We proceed by induction on $n$, noting that the cases $n=0,1$ can be easily verified. Suppose that $n \geq 2$. Given $m>0$ and $g \in C^{m}(A, M), b \in A$, we define

$$
\begin{aligned}
g^{b}\left(a_{1}, \ldots, a_{m}\right)= & g\left(a_{1}, \ldots, a_{m-1}, a_{m} b\right)-g\left(a_{1}, \ldots, a_{m}\right) \cdot b \\
& -g\left(a_{1}, \ldots, a_{m-1}, b\right) \cdot a_{m}
\end{aligned}
$$

as well as

$$
g_{b}\left(a_{1}, \ldots, a_{m-1}\right)=g\left(a_{1}, \ldots, a_{m-1}, b\right) .
$$

Directly from the definitions we obtain the identities

$$
\begin{gathered}
\delta(g)_{b}=\delta\left(g_{b}\right)+(-1)^{m} g^{b}, \quad g \in C^{m}(A, M), \\
\left(\gamma_{a}(g)\right)_{b}=\gamma_{a}\left(g_{b}\right)+(-1)^{m}\left(g_{a}\right)_{b}, \quad g \in C^{m+1}(A, M) .
\end{gathered}
$$

Now suppose that $f \in C^{n}(A, M)$. It evidently suffices to show that

$$
\left(\delta\left(\gamma_{a}(f)\right)\right)_{b}+\left(\gamma_{a}(\delta(f))\right)_{b}=\mu_{a}(f)_{b} \quad \forall a, b \in A .
$$

From (1) and (2) we conclude

$$
\begin{aligned}
\delta\left(\gamma_{a}(f)\right)_{b} & =\delta\left(\gamma_{a}(f)_{b}\right)+(-1)^{n-1} \gamma_{a}(f)^{b} \\
& =\delta\left(\gamma_{a}\left(f_{b}\right)+(-1)^{n-1}\left(f_{a}\right)_{b}\right)+(-1)^{n-1} \gamma_{a}(f)^{b},
\end{aligned}
$$

while

$$
\begin{aligned}
\gamma_{a}(\delta(f))_{b} & =\gamma_{a}\left(\delta(f)_{b}\right)+(-1)^{n}\left(\delta(f)_{a}\right)_{b} \\
& =\gamma_{a}\left(\delta\left(f_{b}\right)+(-1)^{n} f^{b}\right)+(-1)^{n}\left(\delta(f)_{a}\right)_{b} .
\end{aligned}
$$

The inductive hypothesis yields

$$
\delta\left(\gamma_{a}\left(f_{b}\right)\right)+\gamma_{a}\left(\delta\left(f_{b}\right)\right)=\mu_{a}\left(f_{b}\right) .
$$

\footnotetext{
${ }^{1}$ Note added in proof. After this was written, the author became aware of the fact that $1.1 \mathrm{can}$ be obtained from Proposition 6.1 of [2, p. 278].
} 
We combine this with the above identities in order to obtain

$$
\begin{aligned}
\left(\delta \circ \gamma_{a}+\gamma_{a} \circ \delta\right)(f)_{b}= & \mu_{a}\left(f_{b}\right)+(-1)^{n-1}\left(\gamma_{a}(f)^{b}-\gamma_{a}\left(f^{b}\right)\right) \\
& +(-1)^{n}\left(\left(\delta(f)_{a}\right)_{b}-\delta\left(\left(f_{a}\right)_{b}\right)\right) .
\end{aligned}
$$

Applying (1) to $f_{a}$ we obtain

$$
\begin{aligned}
\left(\delta(f)_{a}\right)_{b}-\delta\left(\left(f_{a}\right)_{b}\right) & =\left(\delta(f)_{a}\right)_{b}+(-1)^{n-1}\left(f_{a}\right)^{b}-\delta\left(f_{a}\right)_{b} \\
& =\left(\delta(f)_{a}-\delta\left(f_{a}\right)\right)_{b}+(-1)^{n-1}\left(f_{a}\right)^{b} \\
& =(-1)^{n}\left(f^{a}\right)_{b}+(-1)^{n-1}\left(f_{a}\right)^{b}=(-1)^{n-1}\left(\left(f_{a}\right)^{b}-\left(f^{a}\right)_{b}\right) .
\end{aligned}
$$

Since

$$
\begin{aligned}
\left(f_{a}\right)^{b}-\left(f^{a}\right)_{b}\left(a_{1}, \ldots, a_{n-1}\right)= & f\left(a_{1}, \ldots, a_{n-1} b, a\right)-f\left(a_{1}, \ldots, a_{n-2}, b, a\right) \cdot a_{n-1} \\
& -f\left(a_{1}, \ldots, a_{n-1}, b a\right)+f\left(a_{1}, \ldots, a_{n-1}, b\right) \cdot a,
\end{aligned}
$$

while

$$
\begin{aligned}
\gamma_{a}(f)^{b} & -\gamma_{a}\left(f^{b}\right)\left(a_{1}, \ldots, a_{n-1}\right) \\
= & (-1)^{n-1} f\left(a_{1}, \ldots, a_{n-1} b, a\right)-(-1)^{n-1} f\left(a_{1}, \ldots, a_{n-2}, b, a\right) \cdot a_{n-1} \\
& -(-1)^{n-1} f\left(a_{1}, \ldots, a_{n-1}, a b\right)+(-1)^{n-1} f\left(a_{1}, \ldots, a_{n-1}, b\right) \cdot a,
\end{aligned}
$$

we see that the last two summands of $(3)$ equal $f_{[b, a]}$. Consequently

$$
\left(\delta \circ \gamma_{a}+\gamma_{a} \circ \delta\right)(f)_{b}=\mu_{a}\left(f_{b}\right)+f_{[b, a]}=\mu_{a}(f)_{b},
$$

as desired.

Let $M$ be a two sided $A$-module. When convenient, we regard $M$ as a left $\left(A \otimes_{F} A^{\text {opp }}\right)$-module, where

$$
(a \otimes b) \cdot m=(a \cdot m) \cdot b=a \cdot(m \cdot b) .
$$

Of course, $A^{\text {opp }}$ is the opposite algebra of $A$. For a subset $S$ of $A$, we denote by $C_{A}(S)$ the centralizer of $S$ in $A$.

THEOREM 1.2. ${ }^{2}$ Let $S^{*}$ denote the subalgebra without unity of $A \otimes_{F} A^{\text {opp }}$ which is generated by the elements of the form $s \otimes 1-1 \otimes s$ with $s \in S$. Assume there is an element in $S^{*}$ acting invertibly on $M$. Then the restriction image of $H^{n}(A, M)$ in $H^{n}\left(C_{A}(S), M\right)$ is (0) for every $n$.

Proof. We put $B:=C_{A}(S)$ and consider the linear mapping $j: A \rightarrow A \otimes_{F}$ $A^{\text {opp }}, j(a)=a \otimes 1-1 \otimes a$. For every element $u \in A \otimes_{F} A^{\text {opp }}$, let $l_{u}$ denote the multiplication on $M$ effected by $u$. Let $Z^{n}(A, M):=\operatorname{ker} \delta \cap C^{n}(A, M)$ be the space of $n$-cocycles. Since every element of $S$ commutes with all of the elements of $B$, we obtain

$$
\mu_{s}(f)\left(b_{1}, \ldots, b_{n}\right)=l_{j(s)} \circ f\left(b_{1}, \ldots, b_{n}\right) \quad \forall b_{i} \in B \forall f \in Z^{n}(A, M) .
$$

It now follows from (1.1) that

$$
\left.\delta\left(\gamma_{s}(f)\right)\right|_{B^{n}}=\left.l_{j(s)} \circ f\right|_{B^{n}} \quad \forall s \in S \forall f \in Z^{n}(A, M) .
$$

\footnotetext{
${ }^{2}$ Note added in proof. The author was recently informed by Professor Hochschild that a special case of (1.2) can be found in A. Guichardet, Cohomologic des groupes topologiques et des algèbres de Lie Cedic/Fernand Nathan, Paris, 1980, p. 325
} 
The above identity entails in conjunction with $\delta^{2}=0$ that

$$
\begin{aligned}
U:=\left\{u \in S^{*}: \exists \gamma_{u}: Z^{n}(A, M) \rightarrow C^{n-1}(A, M):\right. & \\
& \left.\left.l_{u} \circ f\right|_{B^{n}}=\left.\delta\left(\gamma_{u}(f)\right)\right|_{B^{n}} \forall f \in Z^{n}(A, M)\right\}
\end{aligned}
$$

is a subalgebra of $S^{*}$ containing $j(S)$. A direct computation reveals that for any $h \in C^{n-1}(A, M)$ we have

$$
\left.l_{j(s)} \circ \delta(h)\right|_{B^{n}}=\left.\delta\left(l_{j(s)} \circ h\right)\right|_{B^{n}} \quad \forall s \in S .
$$

Consequently,

$$
\left.l_{u} \circ \delta(h)\right|_{B^{n}}=\left.\delta\left(l_{u} \circ h\right)\right|_{B^{n}} \quad \forall u \in S^{*} .
$$

By assumption there is $u_{0} \in S^{*}$ such that $l_{u_{0}}$ is invertible. Put $g:=l_{u_{0}}^{-1} \circ \gamma_{u_{0}}(f)$. We apply this to $g$ and obtain

$$
\begin{aligned}
\left.f\right|_{B^{n}} & =\left.l_{u_{0}}^{-1} \circ \delta\left(\gamma_{u_{0}}(f)\right)\right|_{B^{n}}=\left.l_{u_{0}}^{-1} \circ \delta\left(l_{u_{0}} \circ g\right)\right|_{B^{n}} \\
& =\left.l_{u_{0}}^{-1} \circ l_{u_{0}} \circ \delta(g)\right|_{B^{n}}=\left.\delta(g)\right|_{B^{n}} .
\end{aligned}
$$

This proves our assertion.

It readily follows that if $B$ is a subalgebra of $C_{A}(S)$, then

$$
H^{n}(A, M) \rightarrow H^{n}(B, M)
$$

is trivial. In particular, $H^{n}(A, M)$ vanishes for every $n$ whenever there exists a central element $c$ of $A$ such that $c \otimes 1-1 \otimes c$ acts invertibly on $M$.

COROLlaRY 1.3. Suppose that $M$ is an irreducible $\left(A \otimes_{F} A^{\mathrm{opp}}\right)$-module and let $c \in C(A)$ be a central element such that $c \otimes 1-1 \otimes c$ acts nontrivially on $M$. Then $H^{n}(A, M)=0$ for every integer $n$.

PROOF. By assumption $l_{c}$ is a nonzero homomorphism of $\left(A \otimes_{F} A^{\mathrm{opp}}\right)$-modules. Hence $l_{c}$ is invertible and our above remarks yield the desired result.

We now specialize our considerations to supplemented algebras. Such an algebra is given by a pair $(A, \varepsilon)$, where $\varepsilon: A \rightarrow F$ is a homomorphism of $F$-algebras. The supplementation map $\varepsilon$ canonically endows every left $A$-module $M$ with the structure of a two sided $A$-module by means of

$$
(a \otimes b) \cdot m:=\varepsilon(b) a \cdot m .
$$

We put $A^{+}:=\operatorname{ker} \varepsilon$ and note that for every $a \in A^{+}$the left multiplication by $a \otimes 1-1 \otimes a$ on $M$ coincides with the mapping $m \rightarrow a \cdot m$. We may therefore restate our results as follows.

COROLlaRY 1.4. Let $B$ be a subalgebra of the supplemented algebra $(A, \varepsilon)$. Let $M$ be a left $A$-module and suppose there is $c \in C_{A^{+}}\left(B^{+}\right)$which acts invertibly on $M$. Then the canonical map $H^{n}(A, M) \rightarrow H^{n}(B, M)$ is trivial.

ProOF. As $B=B^{+}+F \cdot 1$ is contained in the centralizer of $c$ in $A$, the result is an immediate consequence of (1.2) and the above remarks. 
By the same token we obtain:

COROLLARY 1.5. Let $M$ be an irreducible module of a supplemented algebra A. If $C\left(A^{+}\right) \cdot M \neq 0$, then $H^{n}(A, M)=0 \forall n$.

2. Cohomology of Lie algebras. Throughout this section, $L$ will denote a Lie algebra over $F$. According to the Poincaré-Birkhoff-Witt Theorem, $L$ is a sub-Lie algebra of its universal enveloping algebra $U(L)$. Let $\varepsilon: U(L) \rightarrow F$ be the associative extension of the zero map $L \rightarrow F$. The theory of derived functors warrants that the cohomology groups of $L$ with coefficients in an $L$-module $M$ (cf. [3]) are isomorphic to the corresponding groups of the supplemented algebra $(U(L), \varepsilon)$.

The following result, which is a direct consequence of (1.4), was obtained by Dzhumadil'daev for special central elements of the universal enveloping algebra of a finite-dimensional modular Lie algebra.

THEOREM 2.1. Let $K \subset L$ be a subalgebra and $M$ an L-module. Suppose there is $c \in C_{U(L)^{+}}\left(U(K)^{+}\right)$which operates invertibly on $M$. Then the canonical restriction map $H^{n}(L, M) \rightarrow H^{n}(K, M)$ vanishes.

COROllary 2.2. Suppose that $M$ is irreducible and that $C\left(U(L)^{+}\right) \cdot M \neq 0$. Then $H^{n}(L, M)=0$ for every $n$.

As an application we shall retrieve the following classical result.

THEOREM 2.3 (WhITEHEAD). Suppose that $L$ is a semisimple, finite dimensional Lie algebra and assume that $\operatorname{ch}(F)=0$. Let $M$ be a finite dimensional $L$-module such that $L \cdot M=M$. Then $H^{n}(L, M)=0 \forall n$.

PROOF. We first prove the assertion under the assumption of $M$ being irreducible.

According to general structure theory, $L$ decomposes into a Lie algebra direct sum $L=\bigoplus_{i=1}^{n} L_{i}, L_{i} \subset L$ simple ideals. Hence there is $i_{0}$ with $L_{i_{0}} \cdot M=M$. The simplicity of $L_{i_{0}}$ then entails in conjunction with Cartan's Theorem that the restriction $\rho_{0}$ of the representation $\rho: L \rightarrow g l(M)$ to $L_{i_{0}}$ has a nondegenerate trace form. The corresponding Casimir element $c_{\rho_{0}}$ lies in $C\left(U\left(L_{i_{0}}\right)^{+}\right) \subset C\left(U(L)^{+}\right)$. An elementary computation shows that $\operatorname{tr}\left(\rho\left(c_{\rho_{0}}\right)\right)=\operatorname{tr}\left(\rho_{0}\left(c_{\rho_{0}}\right)\right)=\operatorname{dim}_{F} L_{i_{0}}$. Hence $\rho\left(c_{\rho_{o}}\right) \neq 0$ and $(2.2)$ applies.

In the general situation we employ the fact that every finite dimensional $L$ module $M$ is completely reducible. We write $M=\bigoplus_{i=1}^{m} M_{i}, M_{i} \subset M$ irreducible. Then $L \cdot M_{i}=M_{i}$ and $H^{n}(L, M) \cong \bigoplus_{i=1}^{m} H^{n}\left(L, M_{i}\right)=0$.

THEOREM 2.4. Let $\rho: L \rightarrow g l(M)$ be a finite dimensional faithful irreducible representation. Suppose that $H^{n}(L, M) \neq 0$ for some $n \geq 0$. Then $\operatorname{char}(F)>0$ and $L$ is semisimple.

ProOF. Suppose that $\operatorname{char}(F)=0$. By a theorem of Jacobson (cf. [7, p. 44]) there exists a semisimple subalgebra $L^{\prime} \subset L$ such that $L=L^{\prime} \oplus C(L)$, where $C(L)$ denotes the center of $L$. As $C(L) \subset C\left(U(L)^{+}\right)$, our present assumptions entail in combination with $(2.2)$ that $C(L) \cdot M=0$. Hence $C(L) \subset \operatorname{ker} \rho=0$. As $L \cdot M=M$, (2.3) now implies $H^{n}(L, M)=0 \forall n$, a contradiction. 
Let $p>0$ denote the characteristic of $F$ and suppose that $J \subset L$ is an abelian ideal, $x \in J$. Then $(\operatorname{ad} x)^{p}=0$ and $x^{p} \in C\left(U(L)^{+}\right)$. By virtue of $(2.2)$ it follows that $x^{p}$ annihilates $M$. Hence $J$ operates nilpotently on $M$ and Engel's Theorem guarantees that $V:=\{m \in M ; x \cdot m=0 \forall x \in J\}$ is a nonzero submodule of $M$. As $M$ is irreducible, $V$ and $M$ coincide. Thus $J \subset \operatorname{ker} \rho=0$ proving the semisimplicity of $L$.

\section{REFERENCES}

1. D. W. Barnes, On the cohomology of soluble Lie algebras, Math. Z. 101 (1967), 343-349.

2. H. Cartan and S. Eilenberg, Homological algebra, Princeton Univ. Press, Princeton, N.J., 1956.

3. C. Chevalley and S. Eilenberg, Cohomology theory of Lie groups and Lie algebras, Trans. Amer. Math. Soc. 63 (1948), 85-124.

4. J. Dixmier, Cohomologie des algèbres de Lie nilpotentes, Acta Sci. Math. (Szeged) 16 (1955), 246-250.

5. A. S. Dzhumadil'daev, On the cohomology of modular Lie algebras, Math. USSR-Sb. 47 (1984), 127-143.

6. G. P. Hochschild, On the cohomology groups of an associative algebra, Ann. of Math. (2) 46 (1945), $58-67$.

7. J. Jacobson, Lie algebras, Dover, New York, 1979.

8. J. H. C. Whitehead, On the decomposition of an infinitesimal group, Proc. Cambridge Philos. Soc. 32 (1936), 229-237.

9. _ Certain equations in the algebra of a semi-simple infinitesimal group, Quart. J. Math. Oxford 8 (1937), 220-237.

Department of Mathematics, University of CAlifornia, Riverside, CaliforNIA 92521

Current address: Department of Mathematics, North Carolina State University, Raleigh, North Carolina 27695 